\title{
NEAR-INFRARED OBSERVATIONS OF IRAS MINISURVEY GALAXIES
}

\author{
DAVID P. CARICO AND B. T. SOIFER \\ Palomar Observatory, California Institute of Technology, Pasadena, California 91125
}

C. BEICHMAN

Infrared Processing and Analysis Center, California Institute of Technology, Pasadena, California 91125

and

Jet Propulsion Laboratory, Pasadena, California 91125

J. H. Elias, K. Matthews, and G. Neugebauer

Palomar Observatory, California Institute of Technology, Pasadena, California 91125

Received 3 July 1986; revised 26 August 1986

\begin{abstract}
Near-infrared photometry at $J, H$, and $K$ has been obtained for 82 galaxies from the IRAS minisurvey, a sample of infrared-selected galaxies. The near-infrared colors of these galaxies are similar to those of normal field spiral galaxies, but cover a larger range in $J-H$ and $H-K$. There is evidence of a tighter correlation between the near- and far-infrared emission than exists between the far-infrared and the visible emission. These results suggest that hot-dust emission contributes to the $2.2 \mu \mathrm{m}$ luminosity, and extinction by dust affects both the near-infrared colors and the visible luminosities. In addition, there is an indication that the far-infrared emission in many of the minisurvey galaxies is coming from a strong nuclear component.
\end{abstract}

\section{INTRODUCTION}

The IRAS minisurvey contained 86 galaxies that were detected at $60 \mu \mathrm{m}$ and have Galactic latitude $|b|>20^{\circ}$ and visible counterparts on the Palomar Observatory Sky Survey (POSS) (see Soifer et al. 1984, as well as Rowan-Robinson et al. 1984, for an explanation of the IRAS minisurvey). As such, the galaxies in that survey represent a complete farinfrared-selected sample of galaxies. Excess infrared emission in galaxies can be produced through various mechanisms, including (1) dust in the source galaxy which preferentially absorbs short-wavelength radiation, with reemission by dust of the absorbed starlight; this re-emission can be either in the far infrared, for cold interstellar dust, or the near infrared, for hot circumstellar dust, and (2) nonthermal emission in the source galaxy. In order to study the general characteristics of far-infrared-bright galaxies in the near infrared in an effort to better understand the possible source of the infrared luminosity in such galaxies, this paper presents near-infrared observations of the IRAS minisurvey galaxies and, utilizing also the available $I R A S$ data, provides an analysis of the near- and far-infrared properties of the minisurvey sample of far-infrared-bright galaxies.

\section{OBSERVATIONS AND DATA REDUCTION}

\section{a) Near-Infrared Observations}

The 86 galaxies analyzed by Soifer et al. (1984) which comprise the minisurvey sample are listed in IRAS Circular No. 6 (1984). Of these, 82 were observed at $1.25 \mu \mathrm{m}(J)$, $1.65 \mu \mathrm{m}(H)$ and $2.2 \mu \mathrm{m}(K)$ using the $5 \mathrm{~m}$ Hale telescope at Palomar Observatory; these galaxies are listed in Table I. A solid-nitrogen-cooled InSb detector was used for the observations, and for most of the galaxies a $10^{\prime \prime}$-diameter beam was used. There were three observations (of $17153+1141$, $17152+1940$, and $17223+1906)$ which used a $5 "$-diameter beam, and one (of $18064+3942$ ) which used an 8" diameter beam; possible beam-size effects are discussed below. Sky subtraction was achieved by chopping to reference positions 15 " north and south of the galaxy. The statistical uncertainties in the observed magnitudes are $<0.08 \mathrm{mag}$, and are due primarily to errors in centering the beam on the nucleus of the galaxy. Since the corresponding colors are relatively insensitive to the beam position, their statistical uncertainties are $<0.04 \mathrm{mag}$.

There were four galaxies from the original sample of Soifer et al. (1984) which were not observed in the near infrared. Of these, one galaxy, $18056+3533$ $(1805+356 \mathrm{P06})$, is too large for a measurement appropriate for this analysis to be made with the $5 \mathrm{~m}$ telescope. The remaining three are discussed below (Sec. II $b$ ).

For several of the IRAS sources, there was more than one object within a $3 \sigma$ uncertainty radius $\left(\sim 1^{\prime}\right)$ of the IRAS position. In such cases, near-infrared measurements were taken for all nearby objects which were not obviously pointlike, and various methods were subsequently used to associate a single object with the $I R A S$ source. For some cases, the near-infrared colors showed objects to be foreground stars. In other cases, accurate radio positions (S. Eales 1986) were used to identify the $I R A S$ source on the assumption that the radio source and the IRAS source are the same (see, for example, Helou, Soifer, and Rowan-Robinson 1985; and Sanders and Mirabel 1985). When radio positions were not available, the object which was closest in position to the $I R A S$ source was taken to be the infrared source, provided that it was at least comparable in size and brightness to the other nearby object(s). For cases in which a single galaxy could not be selected positionally, the galaxy which was significantly brighter at $2.2 \mu \mathrm{m}$ was chosen. If no unambiguous determination could be made, positionally or otherwise, the flux densities at each wavelength for each nonstellar object within 1' of the IRAS position were added together. The cases where multiple objects were found near the IRAS position are listed in the notes to Table I.

Heliocentric redshifts have been obtained or were available in the literature for 80 of the 82 galaxies that were observed in the near infrared. Most of these redshifts were obtained using either the double spectrograph (Oke and Gunn 


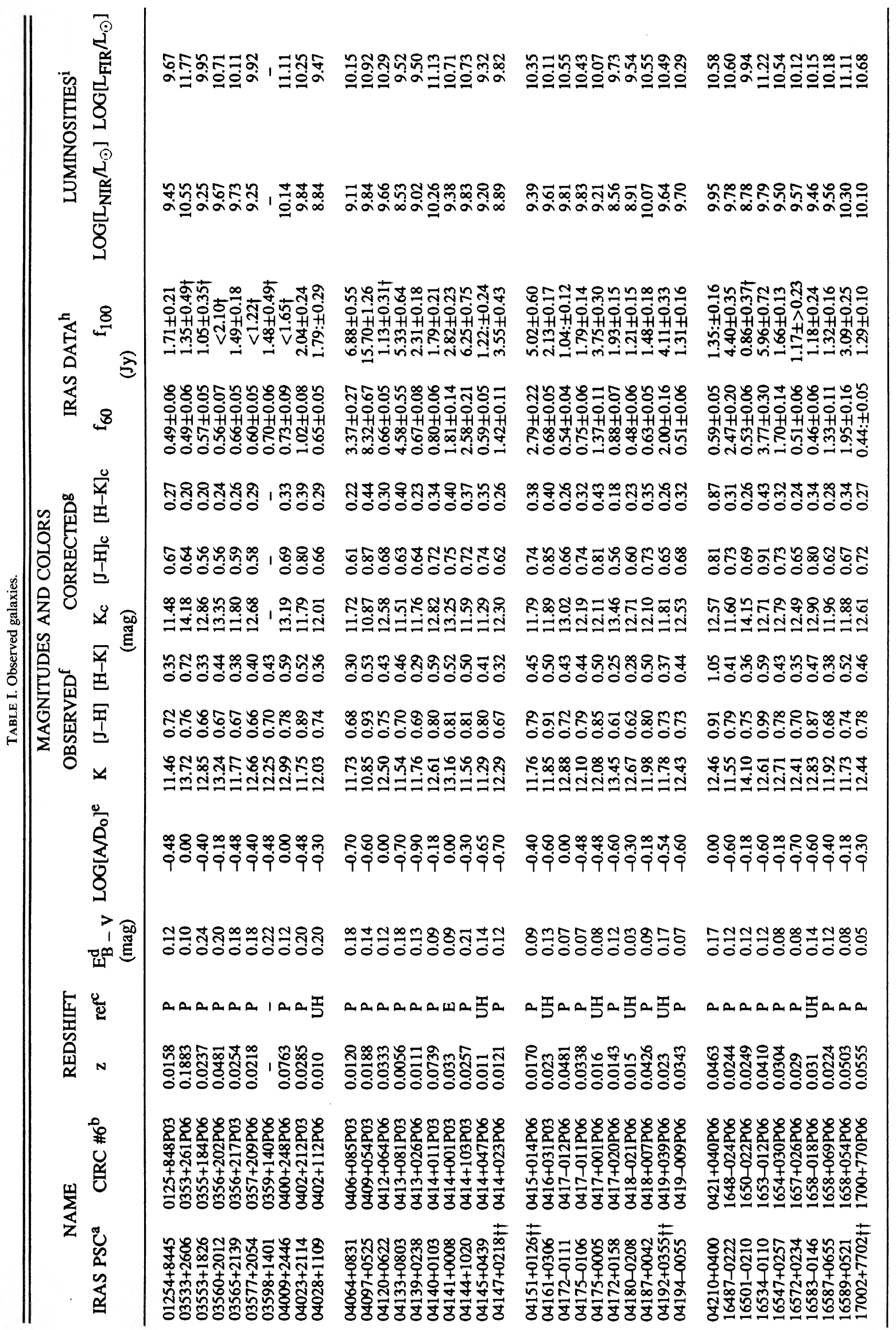




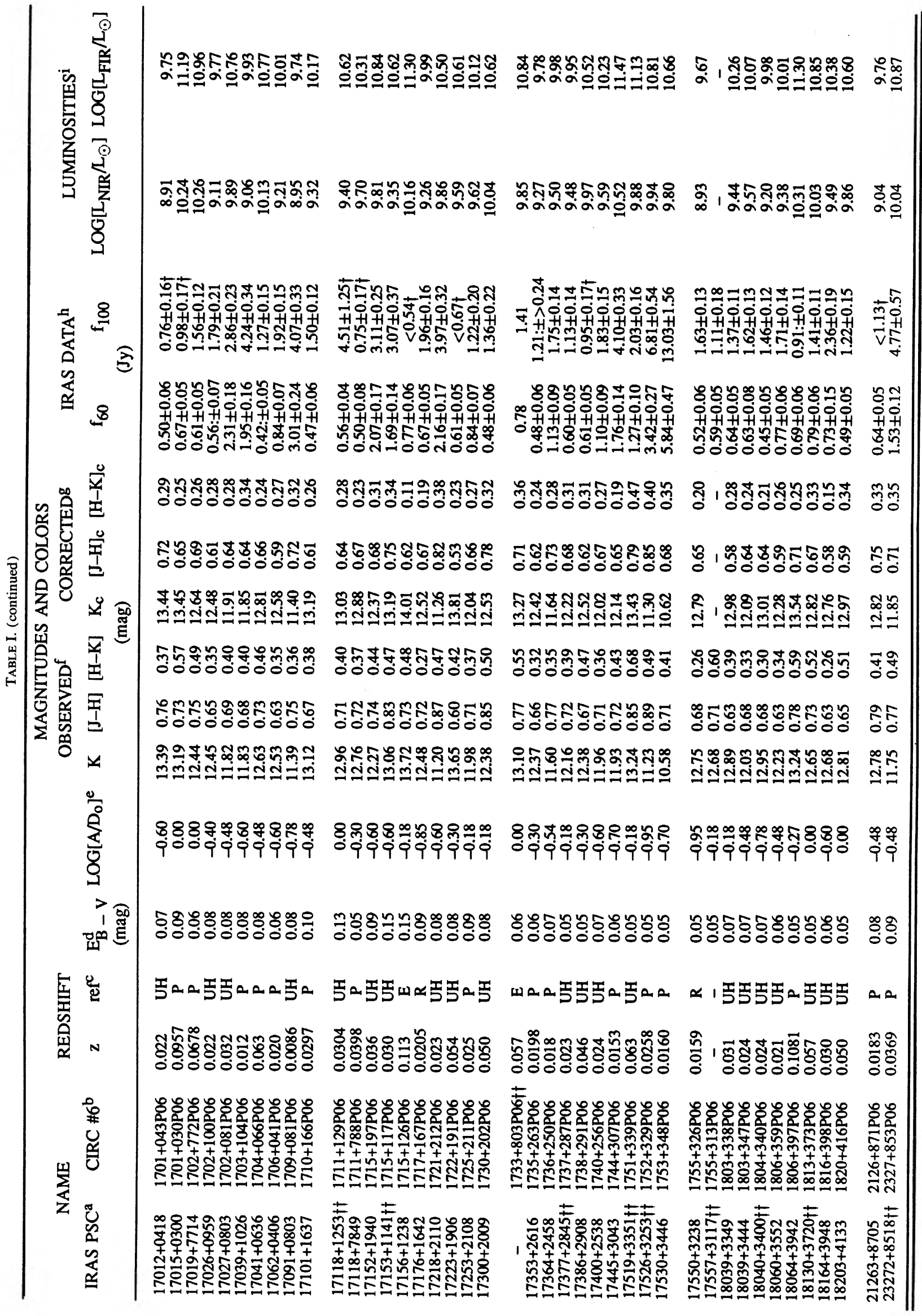


a Infrared Astronomical Satellite (IRAS) Catalogs and Atlases: The Point Source Catalog (1985)

(1984). Astron Astrophys. 131, C1.

${ }^{c}$ Reference for redshifts: P = Palomar Observatory (Persson and Persson 1986); UH = University of Hawaii Institute for Astronomy (Becklin, Heasley, Hill, and Wynn-Williams 1986); E = Elston et al. (1985); $\mathrm{R}=\mathrm{Rood}(1980)$

d The color excess taken from the reddening maps of Burstein and Heiles (1982).

"The logarithm of the ratio of the beam size to the galaxy size. $A$ is the beam diameter (generally $10^{\prime \prime}$; see the text) and $D_{0}$ is the galaxy major-axis diameter as determined from the Palomar Observatory Sky Survey blue prints by visually estimating the radius at which the galaxy brightness equals the sky background. ${ }^{f}$ The values quoted have been corrected for wavelength-dependent distortions in the beam profiles (see the text). The statistical uncertainties in the observed magnitudes and colors are $<0.08 \mathrm{mag}$ and $<0.04 \mathrm{mag}$, respectively.

${ }^{\mathrm{g}}$ Corrected for redshift and Galactic reddening.

${ }^{\mathrm{h}}$ From the IRAS Point Source Catalog (PSC) 1985. The uncertainties quoted are calculated from the largest values for each uncertainty range given in the PSC. The symbol $\pm>$ indicates that the quoted uncertainty is a lower limit. A colon following the flux density refers to a "moderate quality" measurement; no colon indicates a "high quality" measurement. See the IRAS Expanatory Supplement (1985) for a complete discussion of the uncertainties and quality of the IRAS measurements. Flux densities obtained from co-addition of $I R A S$ survey data are indicated by $\dagger$; cases where only an improved limit could be obtained are indicated by $<$.

${ }^{i} L_{\mathrm{NIR}}$ and $L_{\mathrm{FIR}}$ are the near- and far-infrared luminosities, respectively, as defined in the text, and $L_{\odot}$ is the solar luminosity.

Notes to TABLE I. (Denoted by $\dagger \dagger$ in table, offset positions given here are only approximate.)

$04147+0218$. There are two galaxies in the field. The galaxy chosen as the minisurvey galaxy lies within $5^{\prime \prime}$ of the IRAS position, and is almost 2 magnitudes brighter at $K$ and considerably larger than the second galaxy, which is $20^{\prime \prime} \mathrm{E}$ and $60^{\prime \prime} \mathrm{N}$ of the IRAS position.

$04151+0126$. There is a foreground star lying $40^{\prime \prime} \mathrm{E}, 15^{\prime \prime} \mathrm{N}$ of the IRAS position. The galaxy is $90^{\prime \prime} \mathrm{W}, 60^{\prime \prime} \mathrm{S}$ of the IRAS position.

$04192+0355$. There are two nonstellar nuclei within a few arcseconds of the IRAS position; their near-infrared flux densities have been added.

$17002+7702$. There are three objects visible near the $I R A S$ position. One at $20^{\prime \prime} \mathrm{W}, 15^{\prime \prime} \mathrm{S}$, is a foreground star. The other two, at $0^{\prime \prime} \mathrm{E}, 15^{\prime \prime} \mathrm{N}$ and $20^{\prime \prime}$ $\mathrm{W}, 0^{\prime \prime} \mathrm{N}$, are nonstellar; the source at $20^{\prime \prime} \mathrm{W}, 0^{\prime \prime} \mathrm{N}$ was chosen as the IRAS source based on the radio-source position (Eales 1986).

$17118+1253$. There are two nonstellar objects, one at $60^{\prime \prime} \mathrm{W}, 20^{\prime \prime} \mathrm{N}$ and a second at $50^{\prime \prime} \mathrm{W}, 0^{\prime \prime} \mathrm{N}$; the latter was chosen as the minisurvey galaxy since it is $1.6 \mathrm{mag}$ brighter at $K$, and its position agrees with the radio-source position (Eales 1986).

17153+1141. A foreground star lies 50" E, 20" S of the IRAS position. The minisurvey galaxy is at $15^{\prime \prime} \mathrm{W}^{\prime \prime}, 0^{\prime \prime} \mathrm{N}$, with a second foreground star lying to the immediate northwest.

1733 +803P6. This galaxy was not included in the IRAS Point Source Cata $\log$. However, the IRAS flux densities for this galaxy were considered valid despite their exclusion from the PSC (see the text). The 60 and $100 \mu \mathrm{m}$ flux densities have been taken as the average of those quantities for the IRAS sources $17329+8016$ and $17331+8016$ from the IRAS working-survey data base.
$17377+2845$. The minisurvey galaxy is at $5^{\prime \prime} \mathrm{E}, 25^{\prime \prime} \mathrm{N}$, with a foreground star lying to the immediate southwest.

$17519+3351$. The minisurvey galaxy is $0^{\prime \prime} \mathrm{E}, 0^{\prime \prime} \mathrm{N}$; there is a second galaxy at $10^{\prime \prime} \mathrm{W}, 60^{\prime \prime} \mathrm{S}$

17526 +3253 . There are two nonstellar nuclei within a few arcseconds of the IRAS position; both nuclei correspond to radio-source positions (Eales 1986). The near-infrared flux densities for both sources have been added together.

$17557+3117$. There are two galaxies in the field, one at $20^{\prime \prime} \mathrm{E}, 0^{\prime \prime} \mathrm{N}$ and another at $10^{\prime \prime} \mathrm{E}, 5^{\prime \prime} \mathrm{N}$. They are of comparable size and brightness, and both galaxies correspond to radio-source positions (Eales 1986). The nearinfrared flux densities for both galaxies have been added together.

$18040+3400$. There are two nonstellar nuclei within a few arcseconds of the IRAS position; their near-infrared flux densities have been added together.

$18130+3720$. There are two galaxies in the field, one at $5^{\prime \prime} \mathrm{W}, 0^{\prime \prime} \mathrm{N}$ and another at $10^{\prime \prime} \mathrm{W}, 20^{\prime \prime} \mathrm{N}$. They are of comparable size and brightness, and their near-infrared flux densities have been added together. There is also a foreground star at $10^{\prime \prime} \mathrm{W}, 15^{\prime \prime} \mathrm{N}$.

$23272+8518$. There are two galaxies in the field, one at $10^{\prime \prime} \mathrm{E}, 0^{\prime \prime} \mathrm{N}$ and another at $5^{\prime \prime} \mathrm{W}, 15^{\prime \prime} \mathrm{N}$. They are of comparable size and brightness, and their near-infrared flux densities have been added together. There is also a foreground star at $30^{\prime \prime} \mathrm{W}, 0^{\prime \prime} \mathrm{N}$.
1982) mounted at the Cassegrain focus of the Palomar $5 \mathrm{~m}$ telescope (Persson and Persson 1986), or the Grism spectrometer on the $2.2 \mathrm{~m}$ telescope at Mauna Kea (Becklin, Heasley, Hill, and Wynn-Williams 1986). The redshifts and their source are listed in Table I.

Corrections were applied to the measured magnitudes of each galaxy to take into account wavelength-dependent distortions in the beam profiles. Two-dimensional representations of the beam shapes for each wavelength were made by combining beam profiles obtained from north-south and east-west scans across a star, and these two-dimensional representations were then numerically convolved with the de Vaucouleurs $r^{1 / 4}$ law (de Vaucouleurs 1948). The scale lengths in the de Vaucouleurs law for each galaxy profile were taken as $D_{0} / 2$, where $D_{0}$ is the galaxy major-axis diameter as determined from the POSS blue prints by visually estimating the radius at which the galaxy brightness equals the sky background. The corresponding corrections subtracted from each observed magnitude were $0.03-0.07 \mathrm{mag}$ in $J$, 0.02-0.05 mag in $H$, and 0.01-0.02 mag in $K$, with uncertainties of $0.01-0.02 \mathrm{mag}$. The resulting corrections to the nearinfrared colors caused a decrease of $0.02-0.03 \mathrm{mag}$ in $J-H$ and $0-0.03 \mathrm{mag}$ in $H-K$, with uncertainties in the corrections on the order of $0.03 \mathrm{mag}$.
Corrections for Galactic reddening and redshift ( $K$ corrections) were also applied to the observed magnitudes. The Galactic reddening corrections $A_{\lambda}$ were obtained for each galaxy from the reddening maps of Burstein and Heiles (1982) using the reddening law of Cohen et al. (1981). The $K$ corrections, $K_{\lambda}$, were obtained by linearly interpolating the $K$ corrections of S. E. Persson as given by Neugebauer $e t$ al. (1985). Using the convention that the corrected magnitude $m_{0 \lambda}$ and the measured magnitude $m$ are related by $m_{0 \lambda}=m-A_{\lambda}-K_{\lambda}$, the following mean values for the corrections and their corresponding dispersions were obtained: $A_{J}=0.08 \pm 0.04 \mathrm{mag}, A_{H}=0.05 \pm 0.02 \mathrm{mag}, A_{K}$ $=0.03 \pm 0.01 \mathrm{mag}$, and $K_{J}=0.02 \pm 0.02 \mathrm{mag}, K_{H}$ $=0.00 \pm 0.01 \mathrm{mag}, K_{K}=-0.12 \pm 0.08 \mathrm{mag}$.

No correction for beam size has been made. Table I gives the values of $\log \left(A / D_{0}\right)$ for the minisurvey galaxies, where $A$ is the beam diameter and $D_{0}$ is as described above. Although the individual measurements of $D_{0}$ are crude, they are nevertheless sufficient to indicate any significant effects that may exist in the sample as a whole. No evidence of any correlation between size and near-infrared colors was apparent. The observed and corrected $K$ magnitudes and near-infrared colors are tabulated in Table I. The two galaxies for which redshifts are not available have not been included in any of the follow- 
ing analysis; nevertheless, their observed near-infrared magnitudes and colors have been included in Table $\mathbf{I}$.

Elston et al. (1985) have published $J, H$, and $K$ measurements for eight of the minisurvey galaxies. In almost every case their color measurements are significantly discrepant with the corresponding measurements presented in this paper. For three of the galaxies for which the greatest discrepancy exists $(17218+2110,17519+3351, \quad$ and $17526+3253$ ), we have repeated the observations, and in each case these repeat measurements were in good agreement with the original Palomar results. The discrepancies are not due to different sources being selected as the correct IRAS source since none of the objects observed from Palomar can account for the extreme near-infrared colors reported by Elston et al., and only two of the minisurvey galaxies for which Elston et al. report $J, H$, and $K$ measurements have other objects nearby which could lead to such confusion.

\section{b) IRAS Data}

The IRAS data used for this analysis were taken from the IRAS Point Source Catalog (1985, hereafter referred to as the PSC) and are listed in Table I. The data used by Soifer $e t$ al. (1984) in their analysis involved flux estimates which were revised prior to publication of the PSC. Two of the galaxies present in Soifer's sample, 0418+058P06 and $1751+319$ P06, did not qualify for inclusion in the PSC, and a third galaxy, $04203+0425(0420+044 \mathrm{P} 06)$, has only a limit listed in the PSC for the $60 \mu \mathrm{m}$ flux density. These galaxies have been excluded from this paper. A fourth galaxy from Soifer's sample, $1733+803 \mathrm{P} 06$, was also excluded from the PSC, but in this case the IRAS observations were judged valid (Chester 1985). The 60 and $100 \mu \mathrm{m}$ flux densities from the IRAS working-survey data base for this galaxy are also listed in Table I. The IRAS Explanatory Supplement (1985) includes a complete discussion of the uncertainties in the IRAS data.

Sixteen of the minisurvey galaxies have only limits in the PSC for the $100 \mu \mathrm{m}$ flux density. For these galaxies, which are flagged in Table I, the improved $100 \mu \mathrm{m}$ flux densities or limits have been obtained from co-addition of IRAS survey data.

\section{RESULTS}

\section{a) Near-Infrared Colors}

The near-infrared colors for the minisurvey galaxies are plotted in Fig. 1. There is one galaxy, $04210+0400$, which is distinct from the rest of the sample, with $J-H=0.81$ and $H-K=0.87$. These measurements are appropriate for a galaxy with contributions at $K$ of roughly $90 \%$ Seyfert nucleus and $10 \%$ normal galaxy (Glass 1981), and are consistent with the findings of Beichman et al. (1985), who find a Seyfert 2 emission-line spectrum for this galaxy.

The region in Fig. 1 enclosed by a dashed line is the region occupied by the normal spiral galaxies of Aaronson (1977), converted to the CIT photometric system of Frogel et al. (1978). The Aaronson sample was not intended as a statistically complete sample, but consists of 91 galaxies that were selected on the basis of being bright, well observed at other wavelengths, and covering a wide range of morphological types. Nevertheless, we will use Aaronson's sample as representative of normal field spiral galaxies.

It can be seen from Fig. 1 that a large fraction of the minisurvey galaxies would be well fitted by the normal galaxy

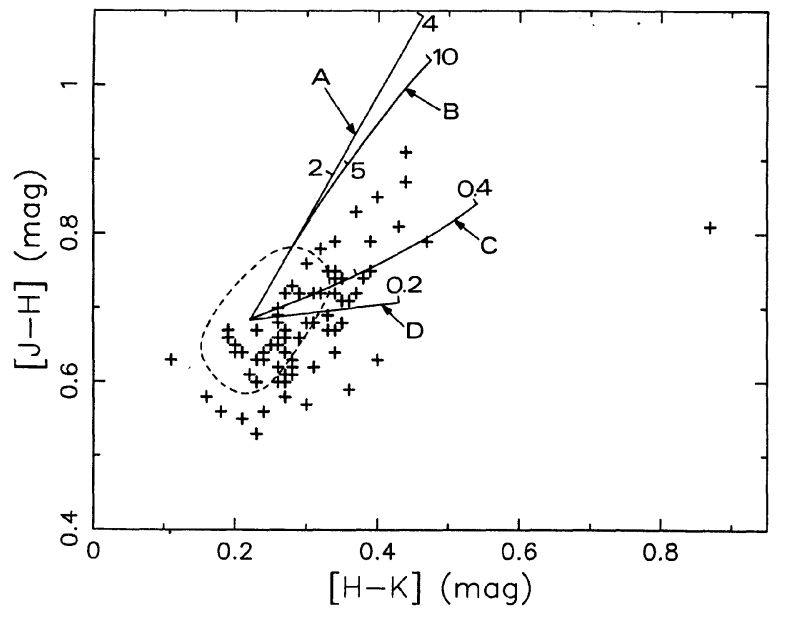

FIG. 1. Near-infrared colors for the IRAS minisurvey galaxies. The region enclosed by a dashed line is that occupied by the normal spiral galaxies of Aaronson (1977). The solid lines A through D represent contributions to the near-infrared colors as follows: A corresponds to a reddening screen of purely absorbing dust, drawn as a function of $\tau_{V}$, the optical depth at $V$; B to reddening from dust that is uniformly mixed with the emitting source, also drawn as a function of $\tau_{V} ; \mathrm{C}, \mathrm{D}$ to 600 and $1000 \mathrm{~K}$ dust, respectively, with emissivity $\epsilon$ varying as $\epsilon \propto v^{2}$, where $v$ is the frequency. Lines $\mathrm{C}$ and $\mathrm{D}$ are labeled according to the fractional contribution to the $2.2 \mu \mathrm{m}$ emission, assuming zero contribution from dust emission for a normal stellar population. All of the lines A through D are taken from Aaronson (1977).

region were it not for a slight shift, amounting to roughly 0.02 mag in both $J-H$ and $H-K$. We consider such a small systematic effect with skepticism in view of the systematic uncertainties inherent in the comparison between the two samples. The effect can also be viewed as a shift of $\sim 0.04$ mag in $H-K$ alone, making it consistent with a contribution to the $2.2 \mu \mathrm{m}$ flux from dust emission. However, a conclusive determination as to whether this shift is a real effect would require measurements of both the minisurvey sample and a set of normal spiral galaxies using the same photometric system and is beyond the limits of this study.

The minisurvey galaxies are redder, on average, than the Aaronson galaxies and show a larger dispersion in $J-H$ and $H-K$. As is indicated in Fig. 1, this can be understood by invoking the presence of dust in the minisurvey galaxies, affecting the near-infrared colors of a normal stellar population through absorption with visual optical depths up to about 5 , coupled with emission by hot dust. The large internal extinction needed to account for these colors is consistent with the considerable internal extinction in a sample of latetype spiral galaxy nuclei studied by Frogel (1985), and with the finding by De Jong et al. (1984) that most IRAS galaxies are late-type spirals.

A possible alternate source for excess reddening in nearinfrared colors is nonthermal infrared continuum emission. To consider this possibility, it would be worthwhile to know what fraction of the minisurvey galaxies may be Seyfert nuclei. The spectral indices between 60 and $100 \mu \mathrm{m}$ for the minisurvey galaxies have been compared to those in a sample of known Seyfert galaxies studied by Miley, Neugebauer, and Soifer (1985). The spectra of the minisurvey galaxies between 60 and $100 \mu \mathrm{m}$ do appear to be statistically some- 
what steeper than would be expected for Seyfert nuclei, but the difference is only marginally significant and of itself is insufficient to provide a reliable estimate of the Seyfert contribution to the minisurvey sample. De Grijp et al. (1985) have shown that the selection criterion $0.5<\alpha(60,25)<1.25$ (where $\alpha$ is defined by $f_{v} \propto v^{-\alpha}$ ) is a very efficient means of finding previously unknown Seyfert galaxies (see also Osterbrock and De Robertis 1985). Unfortunately, only 21 of the minisurvey galaxies have firm IRAS detections at $25 \mu \mathrm{m}$. Nevertheless, for those 21 galaxies the median spectral index is $\alpha(60,25)=2.1$, which can be compared to the results of Miley, Neugebauer, and Soifer (1985), who find median 60 to $25 \mu \mathrm{m}$ spectral indices for Seyfert 1 and Seyfert 2 galaxies of 1.0 and 1.2, respectively. Although not conclusive, this suggests that the minisurvey probably does not contain a substantial Seyfert population.

It may be possible to identify Seyfert nuclei based on their near-infrared properties. Balzano and Weedman (1981), by analyzing samples of Seyfert, emission-line, and bright compact nuclei, conclude that a value of $J-K>1.1$ mag for a galaxy nucleus indicates an $80 \%$ probability that the nucleus will show indicators of a nonthermal source. The results of Balzano and Weedman, however, are based in large part on an analysis of known Seyfert nuclei, for which the near-infrared colors tend to have a larger $H-K$ than do the minisurvey galaxies. Ten of the minisurvey galaxies have $J-K>1.1 \mathrm{mag}$, but, because the distribution of the minisurvey galaxies corresponds well with the effect of internal extinction from dust absorption, we believe that the nonthermal continuum does not contribute substantially to the nearinfrared colors of the minisurvey galaxies. Nevertheless, this possibility cannot be entirely ruled out based on the data in this paper.

\section{b) Near-and Far-Infrared Luminosity Correlation}

The near- and far-infrared luminosities are tabulated in Table I for all of the galaxies for which redshifts are available. For these calculations, the near-infrared flux is taken as $F_{\text {NIR }}=v f_{v}(2.2 \mu \mathrm{m})$, while the far-infrared flux is taken as the flux between 42 and $122 \mu \mathrm{m}$ (see Appendix B of Cataloged Galaxies and Quasars Observed in the IRAS Survey (1985)). The luminosities were calculated for $H_{0}=75 \mathrm{~km}$ $\mathrm{s}^{-1} \mathrm{Mpc}^{-1}$ and $q_{0}=1 / 2$. The distribution of near-infrared luminosities has a mean $\log \left(L_{\mathrm{NIR}} / L_{\odot}\right)=9.6\left(\right.$ where $L_{\odot}$ is the solar luminosity) with a dispersion of $\log \left(L_{\mathrm{NIR}} / L_{\odot}\right)$ of $\sigma_{\mathrm{NIR}}=0.5$, and the far-infrared luminosity distribution has a mean $\log \left(L_{\mathrm{FIR}} / L_{\odot}\right)=10.4$ with a dispersion of $\log \left(L_{\text {FIR }} / L_{\odot}\right)$ of $\sigma_{\text {FIR }}=0.5$.

Figure 2(a) shows the distribution of $\log \left(L_{\mathrm{FIR}} / L_{\mathrm{NIR}}\right)$ for the minisurvey galaxies; the distribution has a mean $\log \left(L_{\mathrm{FIR}} / L_{\mathrm{NIR}}\right)=0.8$, and a dispersion of $\log \left(L_{\mathrm{FIR}} / L_{\mathrm{NIR}}\right)$ of $\sigma=0.2$. It would be useful to compare the distributions of $\log \left(L_{\mathrm{FIR}} / L_{\mathrm{NIR}}\right)$ and $\log \left(L_{\mathrm{FIR}} / L_{B}\right)$, where $L_{B}$ is the blue luminosity, for the minisurvey galaxies to determine if the far-infrared luminosity is better correlated with the nearinfrared or the blue luminosity. However, these galaxies have not yet been systematically measured at visible wavelengths; the blue magnitudes used by Soifer et al. (1984) were crude estimates. Soifer et al. (1986), however, have compiled a complete sample of 341 IRAS galaxies which have $60 \mu \mathrm{m}$ flux densities greater than $5 \mathrm{Jy}$, Galactic latitude $|b|>30^{\circ}$, and declination $\delta>-20^{\circ}$. This sample, the IRAS bright galaxy sample, is similar to that observed in the mini-

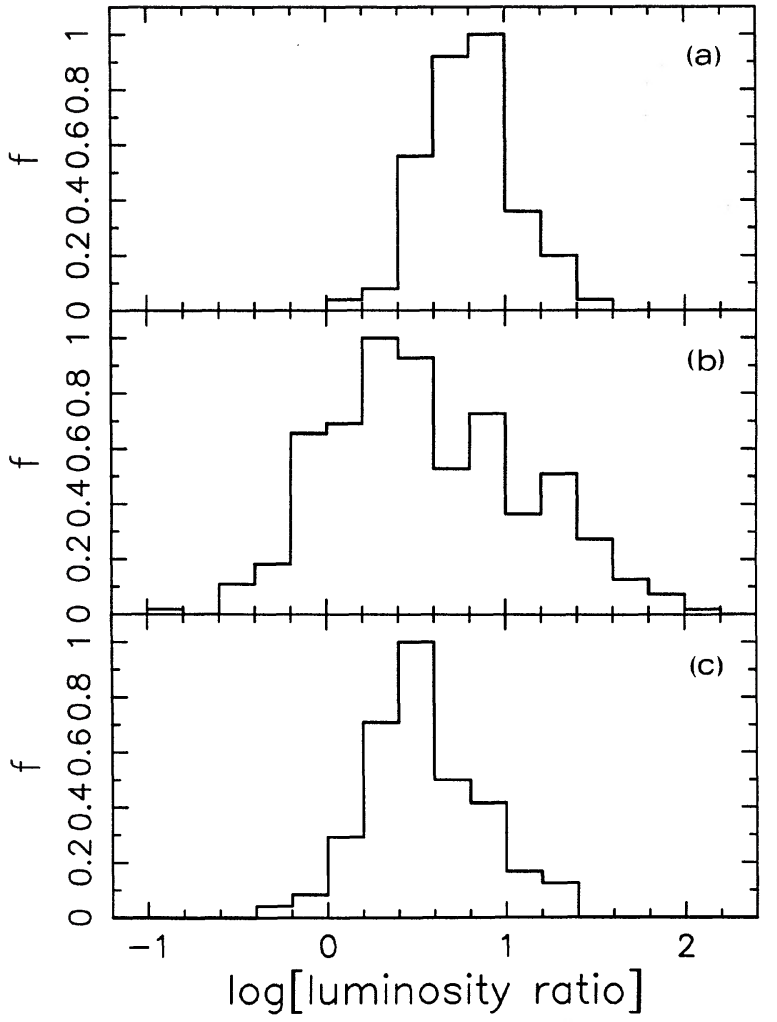

FIG. 2. (a) The distribution of $\log \left(L_{\mathrm{FIR}} / L_{\mathrm{NIR}}\right)$ for the minisurvey galaxies. $L_{\mathrm{FIR}}$ and $L_{\mathrm{NIR}}$ are the far- and near-infrared luminosities, respectively (see the text), and $f$ is the fraction of galaxies, normalized to 1 at the peak in the distribution. (b) The distribution of $\log \left(L_{\mathrm{FIR}} / L_{B}\right)$, where $L_{B}$ is the blue luminosity, for the IRAS bright galaxy sample (Soifer et al. 1986b), normalized to the peak in the distribution. (c) The same as (a), but with the near-infrared luminosities adjusted to $A$ / $D_{0}=1.0$ using the growth curve given by Frogel et al. (1978). $A$ is the beam diameter and $D_{0}$ is the galaxy major-axis diameter as measured on the Palomar Obseratory Sky Survey prints (see the text).

survey in that it represents a complete, flux-limited sample of infrared-selected galaxies; we assume that these galaxies will have similar properties to those in the minisurvey. In Fig. 2(b) the distribution of $\log \left(L_{\mathrm{FIR}} / L_{B}\right)$ for the bright galaxy sample is plotted. It has a dispersion of $\log \left(L_{\mathrm{FIR}} / L_{B}\right)$ of $\sigma_{b g}=0.6$ centered about a mean $\log \left(L_{\mathrm{FIR}} / L_{B}\right)=0.6$.

Clearly, fluctuations and sample size will affect the widths of the distributions. Additional scatter can also be expected in the bright galaxy sample distribution since the blue luminosities for the bright galaxy sample were derived from the blue magnitudes listed in the Zwicky catalog (Zwicky et al. 1961-1968). These effects have been considered by adding an additional uncertainty to the $L_{\mathrm{NIR}}$ measurements, comparable to the uncertainties in the Zwicky magnitudes (which are <0.5 mag; see, for example, Giovanelli and Haynes 1984; or Huchra 1976), and then using an $F$-distribution test to compare the effect of the additional scatter in the minisurvey distribution to the bright galaxy sample distribution. The result is that, to the $99 \%$ confidence level, the quantities $\sigma$ and $\sigma_{b g}$ do not represent dispersions of equivalent parent populations. Thus, to the extent that the minisur- 
vey and the bright galaxy sample provide equivalent representations of far-infrared-bright galaxies, such galaxies do in fact have a tighter correlation between their far- and nearinfrared emission than between their far-infrared and blue light.

Such a tighter correlation in the far-infrared to $2.2 \mu \mathrm{m}$ luminosity again suggests that extinction by dust significantly affects the visible luminosity of far-infrared-bright galaxies. However, an alternate explanation (Frogel 1986) is that a number of minisurvey galaxies have undergone a period of active star formation in the past $10^{8} \mathrm{yr}$. Such enhanced star formation would produce relatively bright optical magnitudes and blue $U B V$ colors, but would not necessarily produce correspondingly high far-infrared emission. Furthermore, the enhanced optical emission would not be well correlated with the near-infrared emission, which is due primarily to older stars. Without more detailed information on the types of galaxies in the minisurvey, it is not possible to distinguish conclusively between these two possible explanations.

In Fig. 2(c), the distribution of $\log \left(L_{\mathrm{FIR}} / L_{\mathrm{NIR}}\right)$ for the minisurvey galaxies is again shown, where the near-infrared luminosities have been corrected to $A / D_{0}=1.0$ using the growth curve given by Frogel et al. (1978); the corresponding corrections to the $K$ magnitudes are typically in the range from 0 to 1 mag. This distribution has a mean $\log \left(L_{\text {FIR }} / L_{\text {NIR }}\right)=0.5$, with a dispersion of $\log \left(L_{\mathrm{FIR}} / L_{\mathrm{NIR}}\right)$ of $\sigma_{0.1}=0.3$. An $F$-distribution test again indicates that the increase in scatter in this distribution relative to the distribution in Fig. 2(a) is statistically significant to the $90 \%$ confidence level, implying that the far-infrared emission in the majority of the minisurvey galaxies may have a strong nuclear component.

The use of the near-infrared colors as an indicator of the far-infrared activity in the minisurvey galaxies has been investigated in the color-color plots of Fig. 3. In each of these plots, the intent has been to identify some parameter involving the far-infrared luminosity which would divide the sample into distinct groups such that the near-infrared colors for each group would be distinguishable. In Fig. 3(a), the galaxies have been divided into three groups according to whether their far-infrared luminosity lies in the upper, middle, or lower range of the far-infrared luminosities for the entire sample. The luminosity cutoff for each group was determined so as to have as nearly equal numbers of galaxies in each group as possible. In Fig. 3(b), a similar division of the sample into three roughly equal-size groups has been done, where the galaxies have been separated by the ratio $L_{\text {FIR }} / L_{\text {NIR }}$. In Fig. $3(\mathrm{c})$, the sample has been separated in a similar manner using the quantity $L_{\mathrm{FIR}} /\left(D_{0}\right)^{2}$, effectively the far-infrared surface brightness. In all cases, the parameters, which should be clear indicators of far-infrared activity, are ineffective in distinguishing the near-infrared colors as a function of the far-infrared emission.

This result, that there is no clear correlation between nearinfrared colors and far-infrared activity, is not surprising if the far-infrared luminosity in these galaxies is not dominated by the emission from the dust which is responsible for the extinction seen in the near infrared. In particular, if the nearinfrared luminosity is extended throughout the disk, as would be expected for a normal stellar population, then the relatively broadbeam near-infrared measurements (with $A$ / $D_{0}>0.2$ for more than $90 \%$ of the sample) will be dominated by disk emission which, considering the above discussion,

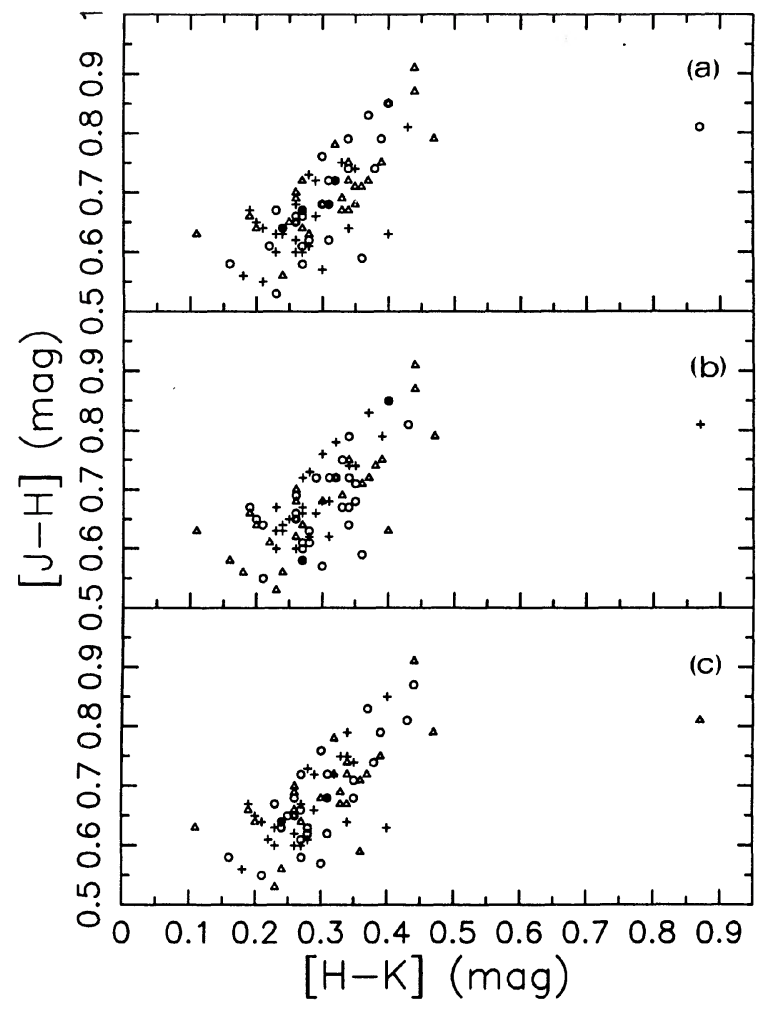

FIG. 3. The near-infrared colors for the minisurvey galaxies. (a) The galaxies are separated according to their far-infrared luminosities, where the symbols $\Delta, O$, and + represent $\log \left(L_{\text {FIR }} / L_{\odot}\right)>10.61$, $10.10<\log \left(L_{\text {FIR }} / L_{\odot}\right)<10.61$, and $\log \left(L_{\text {FIR }} / L_{\odot}\right)<10.10$, respectively. (b) The galaxies are separated according to the ratio of their far-infrared to near-infrared luminosities, where the symbols $\Delta, O$, and + represent $\log \left(L_{\mathrm{FIR}} / L_{\mathrm{NIR}}\right)>0.87,0.64<\log \left(L_{\mathrm{FIR}} / L_{\mathrm{NIR}}\right)$ $<0.87$, and $\log \left(L_{\mathrm{FIR}} / L_{\mathrm{NIR}}\right)<0.64$, respectively. (c) The galaxies are separated according to the effective far-infrared surface brightness, $L_{\mathrm{FIR}} /\left(D_{0}\right)^{2}$, where $D_{0}$ is the galaxy major-axis diameter as measured on the Palomar Observatory Sky Survey blue prints (see the text). The symbols $\Delta, \mathrm{O}$, and + represent $\log \left(L_{\mathrm{FIR}} /\left(L_{\odot} D_{0}^{2}\right)\right)>7.97$, $7.10<\log \left(L_{\mathrm{FIR}} /\left(L_{\odot} D_{0}^{2}\right)\right)<7.96$, and $\log \left(L_{\mathrm{FIR}} /\left(L_{\odot} D_{0}^{2}\right)\right)<7.10$, respectively. For each of the above plots, the cutoffs in the luminosity parameter were determined so as to give roughly equal numbers of galaxies in each of the three groups.

would not necessarily correlate well with the primarily nuclear far-infrared emission.

\section{CONCLUSIONS}

Near-infrared observations of 80 galaxies from the $I R A S$ minisurvey have produced the following results:

(1) The near-infrared colors of far-infrared-bright galaxies are similar to those of normal field spiral galaxies, but show a larger range in $J-H$ and $H-K$ and slightly redder colors, possibly indicating the presence of dust in far-infrared-bright galaxies. For the minisurvey sample, there is evidence for dust absorption of visual optical depths up to about 5, coupled with hot-dust emission.

(2) The far-infrared emission of far-infrared-bright galaxies appears to be more tightly correlated with the near-infrared emission than with the visible emission. This suggests 
the possibility of a significant effect from dust absorption on the visible luminosity of these galaxies, but can also be explained in terms of recent active star formation.

(3) There is an indication that far-infrared-bright galaxies emit a significant fraction of their far-infrared luminosity primarily from a strong nuclear component.

As the manuscript of this paper was being submitted, near-infrared measurements of 22 of the 86 minisurvey galaxies were published by Moorwood, Veron-Cetty, and Glass (1986). Their results are consistent with the results presented in this paper.

The authors extend special thanks to C. G. WynnWilliams, C. J. Persson, and S. E. Persson for helpful sugges- tions on preliminary versions of the manuscript. We also thank E. E. Becklin, J. N. Heasley, G. J. Hill, and C. G. Wynn-Williams of the Institute for Astronomy, University of Hawaii, as well as S. E. Persson of Mount Wilson and Las Campanas Observatories and C. J. Persson of the Infrared Processing and Analysis Center, for generously supplying a number of the redshifts reported in this paper. Steve Eales, also of the Institute for Astronomy, University of Hawaii, provided radio positions to clear up several positional ambiguities. Finally, we gratefully acknowledge a number of useful comments from the referee, Jay Frogel. The research presented in this paper was supported in part by the National Science Foundation grant AST 83-12699 and in part by the NASA IRAS extended mission program.

\section{REFERENCES}

Aaronson, J. (1977). Ph.D. thesis, Harvard University.

Balzano, V. A., and Weedman, D. W. (1981). Astrophys. J. 243, 756

Becklin, E. E., Heasley, J. N., Hill, G. J., and Wynn-Williams, C. G. (1986). Private communication.

Beichman, C., et al. (1985). Astrophys. J. 293, 148

Burstein, D., and Heiles, C. (1982). Astron. J. 87, 1165.

Chester, T. (1985). Private communication.

Cohen, J, G., Frogel, J. A., Persson, S. E., and Elias, J. H. (1981). Astrophys. J. 249, 481.

De Grijp, M. H. K., et al. (1985). Nature 314, 240.

De Jong, T., et al. (1984). Astrophys. J. Lett. 278, L67.

De Vaucouleurs, G. (1948). Ann. Astrophys. 11, 247.

Eales, S. (1986). Private communication.

Elston, R., Cornell, M. E., and Lebofsky, M. J. (1985). Astrophys. J. 296, 106.

Frogel, J. A. (1985). Astrophys. J. 298, 528.

Frogel, J. A., Persson, S. E., Aaronson, M., and Matthews, K. (1978).

Astrophys. J. 220, 75

Frogel, J. A. (1986). Private communication.

Giovanelli, R., and Haynes, M. P. (1984). Astron. J. 89, 1.

Glass, I. S. (1981). Mon. Not. Astron. Soc. 194, 795.

Helou, G., Soifer, B. T., and Rowan-Robinson, M. (1985). Astrophys. J. Lett. 298, L7.

Huchra, J. (1976). Astron. J. 81, 952.

Infrared Astronomical Satellite (IRAS) Catalogs and Atlases: Cataloged Galaxies and Quasars Observed in the IRAS Survey, prepared by C. J. Lonsdale, G. Helou, J. C. Good, and W. Rice (1985). (U.S. GPO, Washington, DC).

Infrared Astronomical Satellite (IRAS) Catalogs and Atlases: The Explanatory Supplement, edited by C. A. Beichman, G. Neugebauer, H. J. Habing, P. E. Clegg, and T. J. Chester (1985). (U.S. GPO, Washington, DC).
Infrared Astronomical Satellite (IRAS) Catalogs and Atlases: The Point Source Catalog (1985). (U.S. GPO, Washington, DC).

Infrared Astronomical Satellite (IRAS) Circular No. 6 (1984). Astron. Astrophys. 131, $\mathrm{Cl}$.

Miley, G. K., Neugebauer, G., and Soifer, B. T. (1985). Astrophys. J. Lett. 293, L11.

Moorwood, A. F. M., Veron-Cetty, M. P., and Glass, I. S. (1986). Astron. Astrophys. 160, 39.

Neugebauer, G., Matthews, K., Soifer, B. T., and Elias, J. H. (1985). Astrophys. J. 298, 275.

Oke, J. B., and Gunn, J. E., (1982). Publ. Astron. Soc. Pac. 94, 586.

Osterbrock, D. E., and De Robertis, M. M. (1985). Publ. Astron. Soc. Pac. 97, 1129.

Persson, S. E., and Persson, C. J. (1986). In preparation.

Rood, H. J. (1980). A Catalog of Galaxy Redshifts (unpublished)

Rowan-Robinson, M., et al. (1984). Astrophys. J. Lett. 278, L7.

Sanders, D. B., and Mirabel, I. F. (1985). Astrophys. J. Lett. 298, L31.

Soifer, B. T., et al. (1984). Astrophys. J. Lett. 278, L71.

Soifer, B. T., et al. (1986). Astrophys. J. Lett. 303, L41.

Zwicky, F., and Herzog, E. (1963). Catalog of Galaxies and Clusters of Galaxies (California Institute of Technology, Pasadena), Vol. 2.

Zwicky, F., and Herzog, E. (1966). Catalog of Galaxies and Clusters of Galaxies (California Institute of Technology, Pasadena), Vol. 3.

Zwicky, F., and Herzog, E. (1968). Catalog of Galaxies and Clusters of Galaxies (California Institute of Technology, Pasadena), Vol. 4.

Zwicky, F., Herzog, E., and Wild, P. (1961 ). Catalog of Galaxies and Clusters of Galaxies (California Institute of Technology, Pasadena), Vol. 1.

Zwicky, F., Karpowicz, M., and Kowal, C. T. (1968). Catalog of Galaxies and Clusters of Galaxies (California Institute of Technology, Pasadena), Vol. 5.

Zwicky, F., and Kowal, C. T. (1968). Catalog of Galaxies and Clusters of Galaxies (California Institute of Technology, Pasadena), Vol. 6. 